\title{
Wet path delay and delay gradients inferred from microwave radiometer, GPS and VLBI observations
}

\author{
Lubomir P. Gradinarsky, Rüdiger Haas, Gunnar Elgered, and Jan M. Johansson \\ Onsala Space Observatory, Chalmers University of Technology, Onsala, Sweden
}

(Received December 25, 1999; Revised June 9, 2000; Accepted June 21, 2000)

\begin{abstract}
Very Long Baseline Interferometry (VLBI) is collocated with a permanent Global Positioning System (GPS) receiver and a Water Vapor Radiometer (WVR) at the Onsala Space Observatory in Sweden. Both space geodetic techniques are affected by the propagation delay of radio waves in the atmosphere, while the remote sensing technique is sensitive to the atmospheric emission close to the center of the $22 \mathrm{GHz}$ water vapor emission line. We present a comparison of estimated equivalent zenith wet delay and linear horizontal delay gradients from an independent analysis of simultaneous VLBI, GPS, and WVR observations. Using different constraints for the variability of the delay and the horizontal gradient in the analysis of the VLBI and the GPS data did not have a large influence on the agreement with the WVR estimates. We found that the weighted rms differences between wet delay estimates from the geodetic techniques and the WVR estimates generally increased for an increased variability in the atmosphere.
\end{abstract}

\section{Introduction}

Variations in the atmospheric refractive index are a major error source for microwave based space geodetic techniques. An azimuthal symmetry and an assumed elevation dependence of the propagation delay have often been used in the VLBI and the GPS processing for estimation of the total zenith delay. It has been shown that azimuthal asymmetries in the atmospheric refractive index will lead to errors in the derived station coordinates (MacMillan, 1995). Including also the estimation of horizontal gradients in the VLBI and the GPS data processing can lead to an improved repeatability of the estimated site coordinates (MacMillan, 1995; Bar-Sever et al., 1998).

The atmospheric parameters derived from space geodetic techniques are not only a disturbing noise for the geodetic applications but are also an important signal for atmospheric studies. Recent investigations concentrate on the possible use of dense permanent GPS networks as multi-purpose networks providing estimates of atmospheric parameters for weather monitoring and prediction (see, e.g. Naito et al., 1998). In order to verify the results for atmospheric parameters derived from the GPS observations it is necessary to compare them to data from independent measurement techniques.

We compare estimated zenith wet delay and linear horizontal delay gradients from the different collocated techniques. We use different constraints for the atmospheric parameters in the geodetic data analysis. We introduce the data, the processing algorithms, the results of the comparisons, and the conclusions.

Copy right $(\mathrm{C}$ The Society of Geomagnetism and Earth, Planetary and Space Sciences (SGEPSS); The Seismological Society of Japan; The Volcanological Society of Japan; The Geodetic Society of Japan; The Japanese Society for Planetary Sciences.

\section{Collocated Techniques}

The Onsala Space Observatory is equipped with collocated remote sensing and space geodetic techniques. The water vapor radiometer (WVR) measures the sky emission at two frequencies $-21.0 \mathrm{GHz}$ and $31.4 \mathrm{GHz}$. The sky emission is caused by the amounts of water vapor, liquid water, and oxygen in the atmosphere. The measured sky brightness temperatures are related to the wet path delay (Elgered, 1993). The WVR operates in a continuous sky scanning mode since 1993.

The $20 \mathrm{~m}$ telescope is a network station in the International VLBI Service for Geodesy and Astrometry (IVS) (Schlüter, 1999). During the period Nov. 93-Oct. 98 Onsala participated in more than 120 geodetic 24 hour long VLBI sessions. The continuously operating GPS receiver is part of the International GPS Service for Geodynamics (IGS).

Since each of the three techniques sometimes had technical problems and the WVR data acquired during rain were discarded, a total of 54 days with simultaneous observations from all three techniques were used.

\section{Atmospheric Parameters of Interest}

The elevation dependencies of the wet and the hydrostatic components of the propagation delay are expressed using mapping functions (Niell, 1996). The zenith value of the hydrostatic part can be estimated with high accuracy based on the ground pressure measurements. Subtracting the hydrostatic component from the total delay yields a wet delay component. A linear azimuthal asymmetry in the troposphere can be described using the model by Davis et al. (1993):

$$
\begin{aligned}
\Delta \tilde{L}^{z}(\epsilon, \phi, \Delta t)= & \Delta L^{z}+V_{L} \Delta t+\left[\Xi_{n} \cos \phi+\Xi_{e} \sin \phi\right] \\
& \times \cot \epsilon\left[1-10^{-6} N_{s} \csc ^{2} \epsilon\right]
\end{aligned}
$$

where $\epsilon$ and $\phi$ are the elevation and the azimuth angles, $\Delta t$ is the time counted from a reference time epoch for which the 
model parameters are estimated, $\Delta \tilde{L}^{z}(\epsilon, \phi)$ is the equivalent zenith wet delay, $\Delta L^{z}$ is the mean zenith delay, $V_{L}$ is the zenith delay rate, $\Xi_{n}$ and $\Xi_{e}$ are north and east horizontal delay gradient components, respectively, and $10^{-6} N_{S} \csc ^{2} \epsilon$ is a correction due to the bending, where $N_{S}$ is the ground refractivity.

The wet delay and the horizontal delay gradient variability can be characterized by using structure functions. We define a temporal structure function of any parameter $\Xi$ as:

$$
\Upsilon_{\Xi}(\tau)=\left\langle[\Xi(t+\tau)-\Xi(t)]^{2}\right\rangle
$$

where $\tau$ is a time lag. Herring et al. (1990) showed that a random walk processes describes the variability of the wet delay reasonably well. For a random walk process $\Upsilon_{\Xi}(1)=$ $\sigma_{r w}^{2}$, where $\sigma_{r w}^{2}$ is the variance of the driving white noise, which scales with the time lag.

We compare the wet delay estimates from WVR observations with those derived from VLBI and GPS data. We use parameter estimates based on 90 minutes of observation, for all the methods. This time was chosen due to the limited number of observations in the VLBI experiments. Note also, that the horizontal delay gradient estimates from the WVR are for the wet part only, whereas the VLBI and the GPS estimates are the total horizontal gradients. We assume that the gradient is due to the wet part, which is not the case when large pressure or temperature gradients exist at the site.

\section{Data Analysis and Results}

The analysis of the WVR data uses an automated editing procedure. Observations obtained within $15^{\circ}$ from the sun are removed. Rain events are detected and the data are removed since the wet delay algorithm brakes down. The gradient model (1) was fit to the data in a least squares sense to estimate the wet delay, the delay rate, and the gradient components.

The VLBI data were processed using the SOLVE software package (Ma et al., 1990) applying the Niell (1996) mapping functions. Earth rotation parameters and radio source coordinates were adopted from a global VLBI solution (Ma and Ryan, 1998). The GPS analysis was made with the GIPSY software package (Webb and Zumberge, 1993). Using the Niell mapping functions, fixed orbits, earth orientation parameters, clock correction parameters, and station coordinates we applied the Precise Point Positioning technique (Zumberge et al., 1997). The constraints for the zenith delay and the horizontal delay gradients used in both VLBI and GPS processing are given in Table 1.

From the time series of zenith wet delay and horizontal delay gradients we identified 8 days with large variations in the atmospheric parameters. For these 8 days we processed the VLBI and the GPS data in 400 different solutions each. The solutions differ in the setup of constraints for the atmospheric parameters used in the software packages (see Table 1). There are 20 different constraints for both the zenith wet delay and the horizontal delay gradients.

As an example, in Figs. 1 and 2, we show the estimated time series of the zenith wet delay and the east horizontal delay gradients for the most variable day. We show only the four extreme "corner solutions" of the VLBI and the GPS
Table 1. Constraint values (in $\mathrm{mm} / \sqrt{h}$ ) for the zenith wet delay and the horizontal delay gradients used in the VLBI and GPS analysis. The characters identify the different solutions.

\begin{tabular}{cccc|cccc}
\hline \multicolumn{3}{c|}{ zenith wet delay } & \multicolumn{4}{|c}{ horizontal delay gradient } \\
\hline $\mathrm{A}$ & 2 & $\mathrm{~K}$ & 17 & $\mathrm{a}$ & 0.2 & $\mathrm{k}$ & 1.7 \\
$\mathrm{~B}$ & 3 & $\mathrm{~L}$ & 20 & $\mathrm{~b}$ & 0.3 & 1 & 2.0 \\
$\mathrm{C}$ & 4 & $\mathrm{M}$ & 23 & $\mathrm{c}$ & 0.4 & $\mathrm{~m}$ & 2.3 \\
$\mathrm{D}$ & 5 & $\mathrm{~N}$ & 27 & $\mathrm{~d}$ & 0.5 & $\mathrm{n}$ & 2.7 \\
$\mathrm{E}$ & 6 & $\mathrm{O}$ & 31 & $\mathrm{e}$ & 0.6 & $\mathrm{o}$ & 3.1 \\
$\mathrm{~F}$ & 7 & $\mathrm{P}$ & 35 & $\mathrm{f}$ & 0.7 & $\mathrm{p}$ & 3.5 \\
$\mathrm{G}$ & 8 & $\mathrm{Q}$ & 40 & $\mathrm{~g}$ & 0.8 & $\mathrm{q}$ & 4.0 \\
$\mathrm{H}$ & 10 & $\mathrm{R}$ & 45 & $\mathrm{~h}$ & 1.0 & $\mathrm{r}$ & 4.5 \\
$\mathrm{I}$ & 12 & $\mathrm{~S}$ & 50 & $\mathrm{i}$ & 1.2 & $\mathrm{~s}$ & 5.0 \\
$\mathrm{~J}$ & 14 & $\mathrm{~T}$ & 56 & $\mathrm{j}$ & 1.4 & $\mathrm{t}$ & 5.6 \\
\hline
\end{tabular}
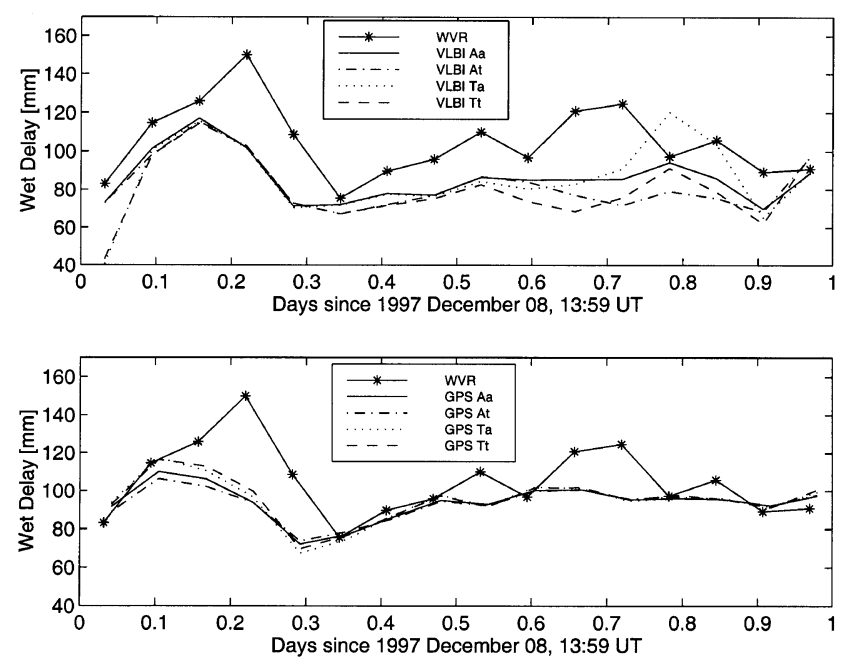

Fig. 1. Time series of estimated zenith wet delay. Shown are WVR results (*) together with results for VLBI (upper plot) and GPS (lower plot). The VLBI and the GPS data display the four "corner solutions" with extreme constraints for the zenith wet delay and the horizontal delay gradients.

results. All other solutions give results that lie between these four extreme solutions.

Both space geodetic techniques give results for the zenith wet delay that follow the WVR results reasonably well. Nevertheless the rapid changes cannot be followed completely with any of the solutions. Overall using different constraints do not impact the agreement between the results very much.

From the time series of the east gradient we see that neither the VLBI data nor the GPS data can follow the variations observes by the WVR when the tightest gradient constraints are used. In the case of VLBI no variations are seen at all. Loosening the gradient constraints enables the space geodetic techniques to sense some of the variations of the gradient results observed by the WVR. The GPS results follow the WVR data better than do the VLBI results. The different zenith wet delay constraints do not affect the gradient results.

The weighted root mean square differences (wrmsd) be- 

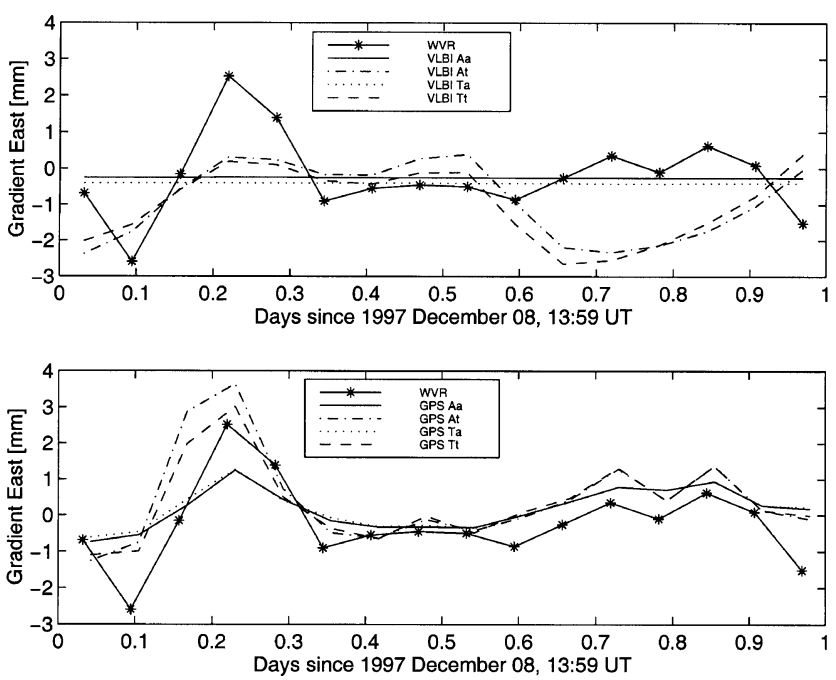

Fig. 2. Time series of estimated east horizontal delay gradients. The WVR results are shown as asterisks together with the VLBI data (upper plot) and the GPS data (lower plot). For both VLBI and GPS the four solutions with extreme constraints are displayed.

tween the WVR results and the results obtained from GPS and VLBI data are displayed in Figs. 3 and 4. Shown are the average wrmsd for the zenith wet delay (WD) and the horizontal delay gradients for the 8 most variable days.

The results for the zenith wet delay indicate that both VLBI and GPS show only a weak dependence of the setup of constraints. The improvement in wrmsd is only $1 \mathrm{~mm}$ from the worst to the best agreement. Nevertheless we can identify relative minima for the wrmsd in the VLBI case which is in the range of $5-15 \mathrm{~mm} / \sqrt{h}$ for the wet delay constraint and $0.5-2 \mathrm{~mm} / \sqrt{h}$ for the gradient constraint. Similar gradient constraints in the GPS case produce a local minima, but it is more difficult to explain the drop in the wrmsd for tight wet delay constraints. The improvement in the agreement between the different techniques, when varying the constraints, is too small to judge on the significance of the impact of the constraints.

Loosening the constraint values further than $20 \mathrm{~mm}$ per $\sqrt{h}$ for the wet delay and $3 \mathrm{~mm} / \sqrt{h}$ for the gradients does not influence the results significantly for any of the techniques. We have also correlated the individual daily variability obtained from WVR data with the values of the constraints that produced the best wrmsd for each of the techniques for each day, but no such correlation was found.

Studying the average wrmsd for the combined gradients in Fig. 4 we see that the choice of the wet delay constraint has no significant impact, only the gradient constraints influence the wrmsd. The wrmsd reaches values between 0.8 and $1 \mathrm{~mm}$ which are of the order of the size of the gradients themselves and illustrates the difficulty to sense these small signals. It is difficult to identify a region of minima for the VLBI solutions, but the "best" region from Fig. 3 gives a small wrmsd here, too. This absence of a clear minimum could be a result of the fact that the VLBI estimates are based on sequential observations of the radio sources and that a tighter constraint is needed in order not to absorb other error sources into the gradient estimates. The typical formal errors for the esti-
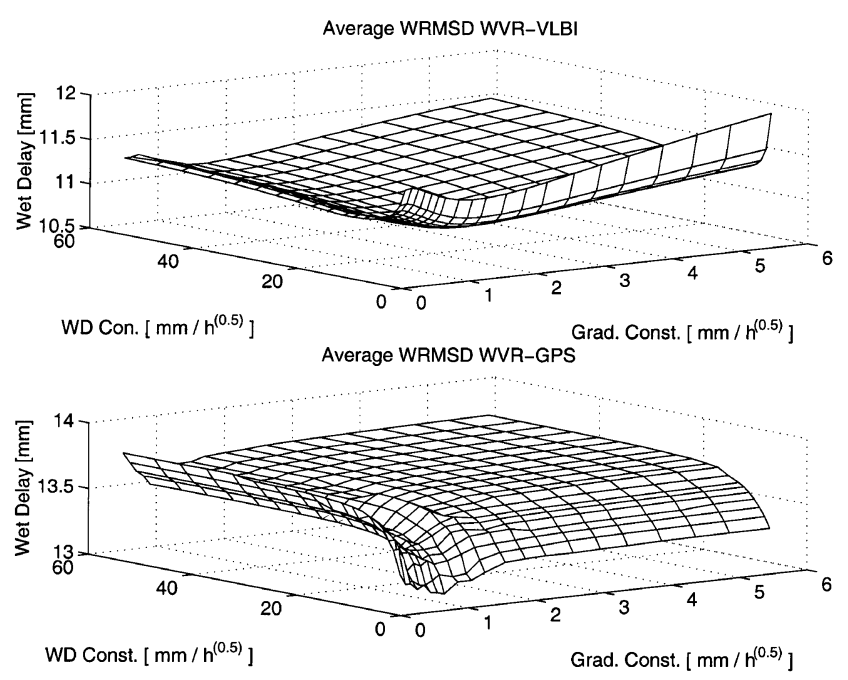

Fig. 3. Average wrmsd for zenith wet delay from all 400 solutions of 8 days with rapid atmospheric variations.
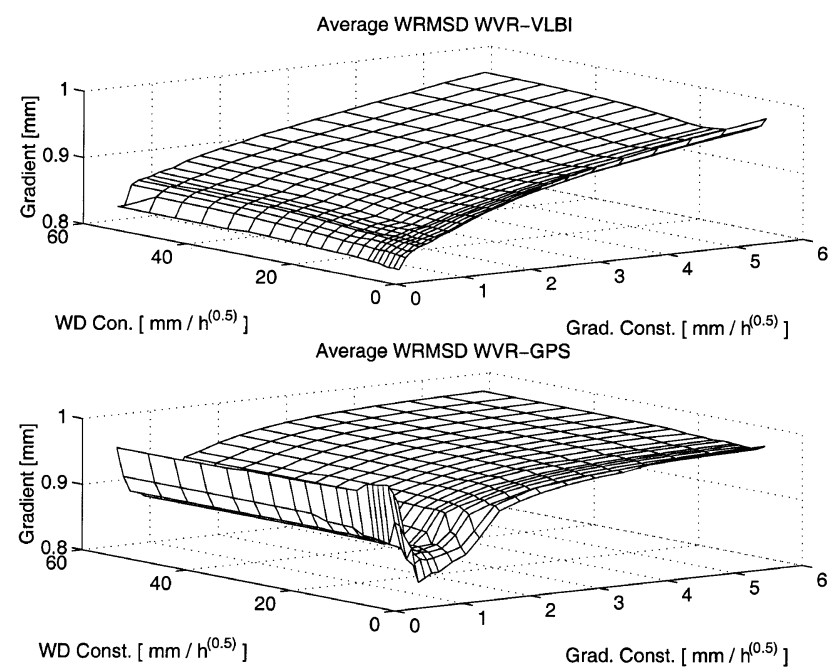

Fig. 4. Average wrmsd for horizontal delay gradients from all 400 solutions of 8 days with rapid atmospheric variations.

mated gradients using VLBI data are 0.09 and $0.40 \mathrm{~mm}$ for the Aa and Tt constraints respectively. For the GPS gradient estimates we have a poor sky coverage in the north given the latitude of Onsala being $57^{\circ} \mathrm{N}$. The corresponding values for the GPS formal errors are however less variable, namely 0.17 and $0.28 \mathrm{~mm}$. The larger variation seen in the VLBI formal errors is due to a varying geometry of the location the observed sources.

Based on these 400 solutions per day for the 8 most variable days in our data set, we ran solutions for all 54 days. For these solutions we used constraints Dd for the GPS solution and Gs for the VLBI solution. The loose gradient constraint in the VLBI case was used in order to be able to detect large values of gradient variability and as we saw previously this is not likely to worsen the over all estimation significantly.

From the WVR data we derived structure functions for the wet delay and for the north and the east gradient component for each day. Figure 5 shows the correlation between the 

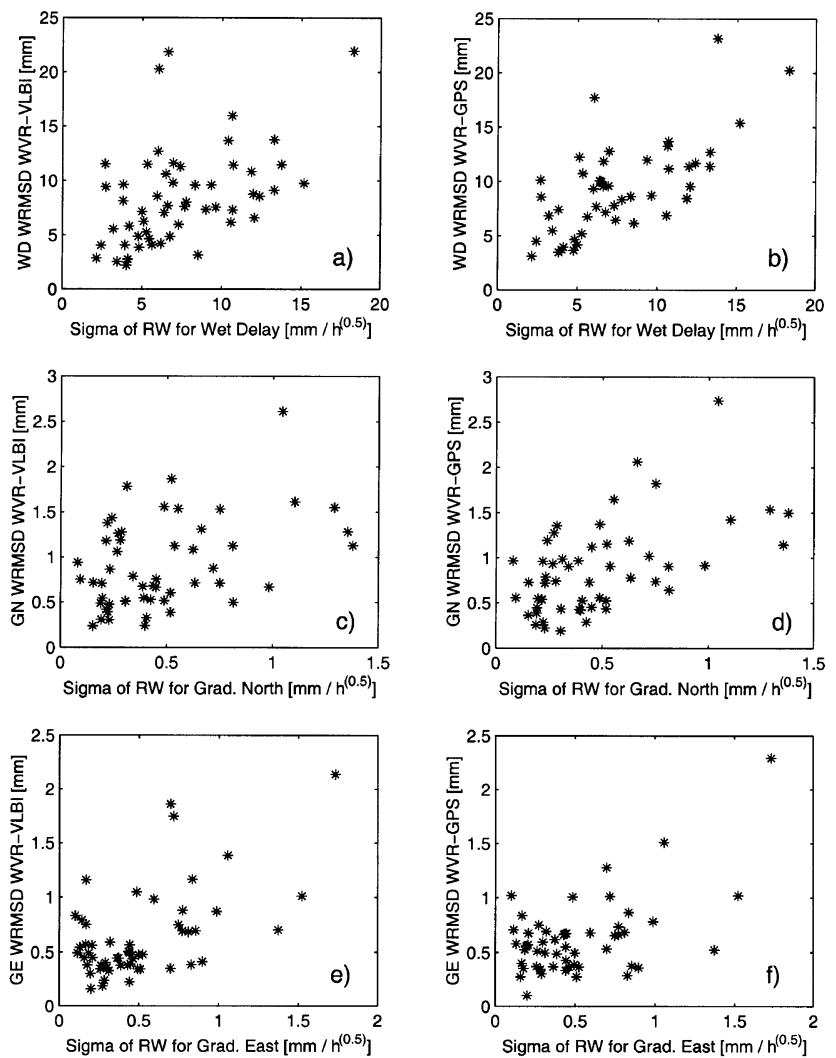

Fig. 5. The wrmsd of VLBI and GPS results with respect to WVR results vs. the sigma of the random walk from structure functions derived from WVR data: a), b) for zenith wet delay, c), d) delay gradients in the north, and $\mathrm{e}), \mathrm{f}$ ) in the east direction.

atmospheric variability inferred from the WVR observations and the wrmsd between the space geodetic techniques and the WVR using all 54 experiments. When the WVR observes a large variability there is also a general increase in the wrmsd.

Finally, correlation analyses were carried out using all data, see Fig. 6. The best agreement for the wet delay estimates is obtained for GPS and VLBI with a wrmsd of $6 \mathrm{~mm}$. The level of agreement between the WVR and GPS or VLBI degrades to $9-10 \mathrm{~mm}$. Figure $6 \mathrm{~d}$ shows the best agreement found for the gradient estimates which was obtained for the east gradient using GPS and VLBI data. In all other cases, when comparing estimated gradients, large discrepancies were obtained.

\section{Conclusions and Future Work}

We analyzed wet delay and horizontal delay gradient estimates derived from 54 days of observations with collocated remote sensing and space geodetic techniques. Based on the 8 most variable days we found that the choice of constraints for the estimated parameters in the space geodetic techniques did not influence the agreement with the WVR estimates significantly. Using all the data we found a dependence of the wrms difference between the estimates obtained from the space geodetic techniques and those from the WVR with respect to the atmospheric variability as derived from the WVR observations. The results for the wet delay are
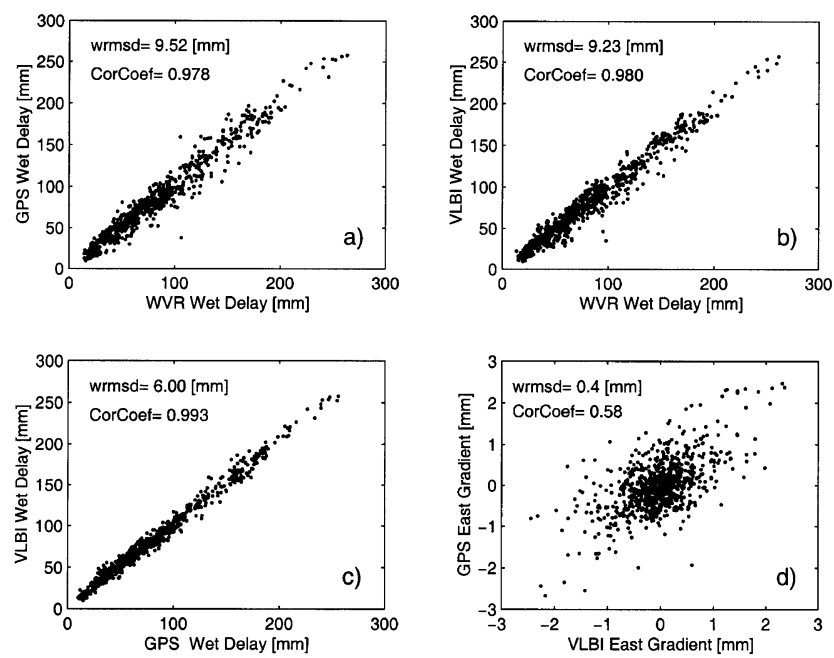

Fig. 6. Correlation plots for the wet delay estimated from WVR, GPS and VLBI a), b), c) and the east gradient estimated from GPS and VLBI d).

highly correlated between the three techniques with the best agreement between VLBI and GPS data. The results for the estimated gradients show much lower correlation, but again the best agreement is between VLBI and GPS data.

\section{References}

Bar-Sever, Y. E., P. M. Kroger, and J. A. Börjesson, Estimating horizontal gradients of tropospheric path delay with a single GPS receiver, $J$. Geophys. Res., 103(B3), 5019-5035, 1998.

Davis, J. L., G. Elgered, A. E. Niell, and C. E. Kuehn, Ground-based measurements of gradients in the "wet" radio refractivity of air, Radio Sci., 28(6), 1003-1018, 1993.

Elgered, G., Tropospheric radio-path delay from ground-based microwave radiometry, in Atmospheric Remote Sensing by Microwave Radiometry, edited by M. A. Janssen, pp. 218-258, Wiley \& Sons, Inc., 1993.

Herring, T. A., J. L. Davis, and I. I.Shapiro, Geodesy by radio interferometry: The application of kalman filtering to the analysis of Very Long Baseline Interferometry data, Geophys. Res. Lett., 95(B8), 12561-12581, 1990.

Ma, C. and J. W. Ryan, NASA Space Geodesy Program-GSFC Data Analysis-1998, VLBI Geodetic Results 1979-1998, August, 1998, http: //lupus.gsfc.nasa.gov/global/glb.html, 1998.

Ma, C., J. M. Sauber, L. J. Bell, T. A. Clark, D. Gordon, and W. E. Himwich, Measurement of horizontal motions in Alaska using very long baseline interferometry, J. Geophys. Res., 95, 21991-22011, 1990.

MacMillan, D. S., Atmospheric gradients from very long baseline interferometry observations, Geophys. Res. Lett., 22(9), 1041-1044, 1995.

Naito, I., Y. Hatanaka, N. Mannoji, R. Ichikawa, S. Shimada, T. Yabuki, H. Tsuji, and T. Tanaka, Global positioning system project to improve Japanese weather, earthquake predictions, EOS Trans. $A G U, \mathbf{7 9}, 301$, 308, 311, June, 1998.

Niell, A., Global mapping functions for the atmosphere delay at radio wavelength, J. Geophys. Res., 101(B2), 3227-3246, 1996.

Schlüter, W., IVS Chairman's Report, International VLBI Service for Geodesy and Astrometry, Annual Report 1999, NASA/TP-1999-209243, 6-9, 1999.

Webb, F. H. and J. F. Zumberge, An introduction to GIPSY/ OASIS-II, JPL Publication D-11088, Pasadena, CA, 1993.

Zumberge, J. F., M. B. Heflin, D. C. Jefferson, M. M. Watkins, and F. H. Webb, Precise point positioning for the efficient and robust analysis of GPS data from large networks, J. Geophys. Res., 102, 5005-5017, 1997.

L. P. Gradinarsky (e-mail: lbg@oso.charmers.se), R. Haas, G. Elgered, and J. M. Johansson 